\title{
Real-time acquisition of multi-view face images to support robust face recognition using a wireless camera network
}

\author{
Srikanth Parupati \\ West Virginia University
}

Follow this and additional works at: https://researchrepository.wvu.edu/etd

\section{Recommended Citation}

Parupati, Srikanth, "Real-time acquisition of multi-view face images to support robust face recognition using a wireless camera network" (2011). Graduate Theses, Dissertations, and Problem Reports. 4764. https://researchrepository.wvu.edu/etd/4764

This Thesis is protected by copyright and/or related rights. It has been brought to you by the The Research Repository @ WVU with permission from the rights-holder(s). You are free to use this Thesis in any way that is permitted by the copyright and related rights legislation that applies to your use. For other uses you must obtain permission from the rights-holder(s) directly, unless additional rights are indicated by a Creative Commons license in the record and/ or on the work itself. This Thesis has been accepted for inclusion in WVU Graduate Theses, Dissertations, and Problem Reports collection by an authorized administrator of The Research Repository @ WVU. For more information, please contact researchrepository@mail.wvu.edu. 


\title{
Real-time acquisition of multi-view face images to support robust face recognition using a wireless camera network
}

\author{
by \\ Srikanth Parupati \\ Thesis submitted to the \\ College of Engineering and Mineral Resources \\ at West Virginia University \\ in partial fulfillment of the requirements \\ for the degree of \\ Master of Science \\ in \\ Electrical Engineering \\ Dr. Arun A. Ross, Ph.D. \\ Dr. David W. Graham, Ph.D. \\ Dr. Vinod Kulathumani, Ph.D., Chair
}

Lane Department of Computer Science and Electrical Engineering

Morgantown, West Virginia

2011

Keywords: Camera network, Multi-view face detection, Face recognition, Buffer management, Opportunistic acquisition, Embedded camera

Copyright 2011 Srikanth Parupati 


\begin{abstract}
Real-time acquisition of multi-view face images to support robust face recognition using a wireless camera network

by

Srikanth Parupati
\end{abstract}

Recent terror attacks, intrusion attempts and criminal activities have necessitated a transition to modern biometric systems that are capable of identifying suspects in real time. But real-time biometrics is challenging given the computationally intensive nature of video processing and the potential occlusions and variations in pose of a subject in an unconstrained environment. The objective of this dissertation is to utilize the robustness and parallel computational abilities of a distributed camera network for fast and robust face recognition.

In order to support face recognition using a camera network, a collaborative middle-ware service is designed that enables the rapid extraction of multi-view face images of multiple subjects moving through a region. This service exploits the epipolar geometry between cameras to speed up multi view face detection rates. By quickly detecting face images within the network, labeling the pose of each face image, filtering them based on their suitability of recognition and transmitting only the resultant images to a base station for recognition, both the required network bandwidth and centralized processing overhead are reduced. The performance of the face image acquisition system is evaluated using an embedded camera network that is deployed in indoor environments that mimic walkways in public places. The relevance of the acquired images for recognition is evaluated by using a commercial software for matching acquired probe images. The experimental results demonstrate significant improvement in face recognition system performance over traditional systems as well as increase in multi-view face detection rate over purely image processing based approaches. 


\section{Acknowledgements}

First and foremost, I would like to express my sincere gratitude to my advisor, Dr.Vinod kulathumani for giving me an opportunity to conduct research on camera sensor networks. It has been privilege to work under him and being a part of his research. His problem solving approach, technical expertise and hard work inspired me to do my best. I always remain grateful to him for supporting throughout my master's program, helping me to improve programming, presentation skills and making those days memorable. This thesis would have not been possible without his insightful suggestions and discussions .

My special thanks to Dr. Arun Ross for helping me to understand the importance of physical interpretation of mathematical equations during pattern recognition course work.

I would like to thank Dr. David W. Graham for his valuable inputs and time.

I would like to acknowledge our research group members Sricharan Ramagiri, Sriram Sankar, Rahul kavi and Rohith Bakkannagari for their support and valuable discussions.

I would like to appreciate Image acquisition toolbox team at The Mathworks Inc for offering an internship opportunity, which exposed me to gain hands on experience with several industrial cameras.

I am always grateful to all of my friends and relatives for their help and support.

Last, but not the least, I am always indebted to my parents and sister for their incredible love, constant support and encouragement to pursue a career of my interest. 


\section{Contents}

$\begin{array}{lll}\text { Acknowledgements } & \text { iii }\end{array}$

List of Figures $\quad$ vi

List of Tables $\quad$ viii

$\begin{array}{ll}\text { Notation } & \text { ix }\end{array}$

1 Introduction $\quad 1$

1.1 Motivation . . . . . . . . . . . . . . . . . . . 1

1.2 Thesis contributions . . . . . . . . . . . . . . . . . 2

1.3 Thesis Outline . . . . . . . . . . . . . . . . . . 4

2 Background information and Related work $\quad 5$

2.1 Background information . . . . . . . . . . . . . . . 5

2.1.1 Related work on multi-view face detection . . . . . . . . . . 7

2.2 Other related work . . . . . . . . . . . . . . . . . . . . . 10

3 Opportunustic face acquisition system $\quad 13$

3.1 System model . . . . . . . . . . . . . . . . . . . . . . . . 13

3.1.1 Assembly of camera platform . . . . . . . . . . . . . . . . 14

3.1.2 Assembly of multi-camera network . . . . . . . . . . . . . . . 15

3.1.3 Software implementation . . . . . . . . . . . . . . 16

3.2 Performance evaluation . . . . . . . . . . . . . . . . . . 18

3.2 .1 Experiment setup . . . . . . . . . . . . . . . . . . . . . . . . . . . . . . 18

3.2.2 Results . . . . . . . . . . . . . . . . . . . . . 19

4 Collaborative multi-view face acquisition system $\quad 23$

4.1 System model . . . . . . . . . . . . . . . . . . . . . . . 23

4.1.1 Epipolar geometry . . . . . . . . . . . . . . 25

4.2 System operation . . . . . . . . . . . . . . . . . . . . 27

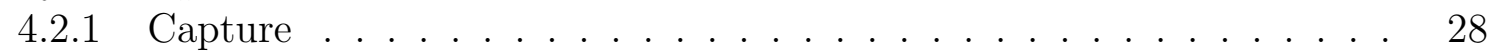

4.2.2 Frontal face detection . . . . . . . . . . . . . . . . . . . . . . . . . . . . . . . . . 28

4.2 .3 Message listening . . . . . . . . . . . . . . . . . . . . . . . . . . . . . . . . . . . . . . 29

4.2 .4 Side face detection $\ldots \ldots \ldots \ldots$. . . . . . . . . . . . . . . . 29 
4.2 .5 Buffer management . . . . . . . . . . . . . . . . . . . . 30

4.3 Performance evaluation . . . . . . . . . . . . . . . . . . . . . . . 33

4.3 .1 Experimental setup . . . . . . . . . . . . . . . 33

4.3 .2 Results . . . . . . . . . . . . . . . . . 34

5 Conclusions and Future work $\quad 36$

5.1 Conclusions . . . . . . . . . . . . . . . . . . . . . 36

5.2 Future work . . . . . . . . . . . . . . . . . 37

$\begin{array}{ll}\text { References } & 38\end{array}$ 


\section{List of Figures}

2.1 Face recognition system . . . . . . . . . . . . . . . .

2.2 Classifier structures for multi-view face detection: Each circle indicates a classifier. The solid arrows are pass route, and the dashed arrows are reject route. (a) Parallel cascade, (b) Detector-pyramid, (c) Decision tree I, (d) Decision tree II . . . . . . . . . . . . . . . . . . . . . . . .

3.1 Smart camera unit: Assembled using a Beagleboard, Logitech 9000 camera and a 802.11 wireless card . . . . . . . . . . . . . . . . . . . . . 14

3.2 Schematics for network based face recognition . . . . . . . . . . . . . 15

3.3 (Top) Deployment of smart camera network with 7 cameras for face recognition experiments. (Bottom) close-up view of individual camera; cameras deployed on 6.5 feet lamp posts . . . . . . . . . . . . . . . . . . .

3.4 Images that did not qualify for transmission to the base station due to: Bad pose (top); motion blur (middle); poor illumination (bottom) . . . . . . . . 17

3.5 Achieved Frame rate vs Capture resolution . . . . . . . . . . . . . . . . . 20

3.6 Correct recognition rate vs number of available cameras . . . . . . . . . . . . 21

3.7 ROC curve of distributed face recognition system with different number of

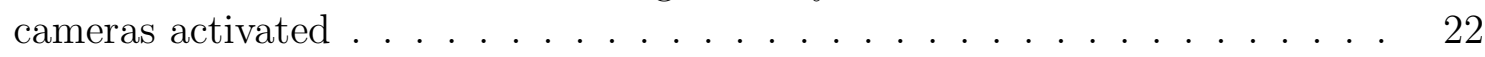

4.1 Our experimental deployment of 3 cameras. The cameras are deployed along an arc of radius 10 feet with a separation of 6 feet between the cameras along the arc as shown. The angles made by the principal axes of cameras $C_{2}$ and $C_{3}$ with that of camera $C_{1}$ are $40^{\circ}$ and $80^{\circ}$ respectively. The cameras are deployed on tripods at a height of 7 feet from the ground. All cameras run a frontal face detector. When a frontal face is detected on any camera, a notification is broadcast to other cameras. . . . . . . . . . . . . . 24

4.2 We classify faces into front, profile and partial profile based on the yaw angles 25

4.3 Point correspondence: The two cameras are indicated by their centers $C$ and $C^{\prime}$ and image planes. The camera centers, 3-D space point $X$, and its image points $x$ and $x^{\prime}$ lie in a common plane . . . . . . . . . . 
4.4 Pseudo-code for operations on each embedded camera. each node executes 4 threads: capture, frontal face detection, message listening and side-face detection. The capture thread samples images at $F$ fps and queues them in $B_{f f}$. The frontal face detection thread dequeues frames from $B_{f f}$ and applies frontal face detector on background subtracted images. If a face is detected, a notification is broadcast to other cameras, otherwise the background subtracted frame is stored in $B_{s f}$. The message listening thread queues any incoming message into $Q$. The side-face detection thread dequeues messages from $Q$, retrieves the synchronous frame corresponding to the message from $B_{s f}$ and performs the side-face detection procedure. . . . . . . . . . . .

4.5 Using camera network geometry for side face detection. When a frontal face is detected on any camera, a notification is broadcast to other cameras specifying the center of the detected face. Epipolar geometry is used to project this point to a corresponding epipolar line (shown as $A B$ ) in the other cameras. The other cameras apply a pose-specific side-face detector in a small region surrounding the segment of the epipolar line that intersects with the background subtracted image. . . . . . . . . . . . . . . . . .

4.6 Example face images detected by our acquisition service. The white rectangles indicate the box enclosing the detected faces in each pose. Face images in each column are extracted from synchronous frames in the three cameras. (a) Images acquired with subjects facing $C_{2}$ : (Top) Frontal face (Middle) Left partial profile face (Bottom) Right partial profile face. (b) Images acquired with subjects facing $C_{1}$ : (Top) Frontal face (Middle) Right partial profile face (Bottom) Right profile face. . . . . . . . . . . . . . . . . 


\section{List of Tables}

3.1 Time taken for various processing operations on the assembled smart camera platform . . . . . . . . . . . . . . . . . 20

4.1 Processing times: Multi-view face detection in clear and cluttered background 34

4.2 Detection rates for frontal and side faces . . . . . . . . . . . . . . . 35 


\section{Notation}

We use the following notation and symbols throughout this thesis.

PTZ - Pan, tilt and zoom

F - Fundamental matrix

l - Epipolar line

Q - Message queue length

NTP - Network time protocol

fps - Frames processed per second

$B_{f f}$ - Frontal face buffer

$B_{s f}$ - Side face buffer

$t_{n d}$ - Network delay in ms

$t_{f f}$ - Average time to detect a face in a background subtracted image in ms

$t_{s f}$ - Average time to detect a side face

$t_{s}$ - Network clock synchronization error in ms

$N_{f f}$ - Frontal face processing rate

$N_{s f}$ - Side face processing rate in fps

$t(x)$ - Time stamp of a frame

$w(x)$ - Width of a detected face

$g_{m}^{i}-M^{t h}$ galley image of subject $i p_{n}-N^{t h}$ probe image of a subject 


\section{Chapter 1}

\section{Introduction}

\subsection{Motivation}

Facial recognition systems have evolved significantly into the most common and reliable mechanism for establishing identity of individuals in the context of applications such as access control, identification of criminals in public places. Traditionally these have been applied for identification from previously acquired photographs, surveillance video tapes that are mostly under controlled environments. Recent terrorist activities and security threats at airports have necessitated a transition to modern facial recognition systems that are capable of identifying suspects in real time. An example scenario is that of raising an alert if any individual from a watch-list database has been detected in the region being monitored, before the subject has moved too far away from the region. But real-time human identification is challenging given the computationally intensive nature of video processing and the potential occlusions and variations in pose of a subject in an unconstrained environment.

Distributed camera networks are ideally suited for such scenarios because they can be deployed to provide coverage from different views of a scene, thus providing tolerance against variable pose, poor illumination and possible occlusions. But realizing such a camera network based human identification system poses the following challenges. (1) Given the bandwidth intensive nature of video data originated from a camera, it is essential to perform local processing within the network before transmitting data to a fusion center. At the same time, a high frame rate of acquisition is required for robust recognition because there may 
be a small duration when a subjects pose is appropriate for recognition and it is desirable to acquire these frames. There is no problem when system is designed to operate in off-line mode based on recorded videos because processing time is not an important factor and all the samples can be used for identification. Hence there is a critical need for distributed algorithms that exploit data obtained from multiple views and simultaneously reduce the processing time and network load.

The objective of this thesis is to support robust face recognition by designing network algorithms for rapid acquisition of multi-view images using a distributed camera network.

\subsection{Thesis contributions}

In order to meet the thesis objective I exploit the following two key insights

- In order to balance the trade-off between local processing at each camera and central computation at the base station, my approach is to use the local resources to perform face detection and to transmit only face images to the base station, by doing so I expect significant reduction in network bandwidth utilization as well as centralized processing overhead in order to maximize the probability of acquiring suitable face image for recognition. The main idea of this approach is to use a densely deployed multi camera network that opportunistically acquire frontal face images of a subject moving through the region covered by the cameras. In order to test the achievable recognition performance using such an opportunistic face acquisition system I have designed embedded camera testbed and used this to perform face recognition in an indoor environments that mimics hallways in public places. Using this set up, overall network bandwidth utilization can be saved up to $90 \%$ by transmitting only face images and it is possible to acquire at least one good face image which is suitable for robust face recognition.

- In unconstrained environments it is not always possible to obtain high quality frontal face images for face recognition. Under such circumstances, non frontal face images acquired from a camera network can be used to improve confidence of face recognition. 
Recent studies[1, 2, 3, 4, 5] have shown that non frontal views and partial views can be used for reliable face recognition, but acquiring multi-view face images in real time is a challenging problem due to computational complexity and diversity in appearance of face images[6]. In this thesis I have designed a collaborative multi-view face acquisition service that uses the geometry of multi-camera network to collaboratively acquire frontal and non-frontal face images in real-time while maintaining high acquisition rate.

The Main idea of the approach is to utilize the multi-view camera geometry and inter camera communication to reduce the computational overhead involved in multi-view face detection. An overview of our approach is as follows: We first train face detectors based on Haar-like features $[7,8]$ for each pose class that is required to be detected and then run a frontal-face detector on each camera in the network. Whenever a frontal face has been detected on any camera, say $C_{f}$, it broadcasts a notification to other cameras to narrow down their search to the region surrounding the epipolar line corresponding to the point where the frontal face is detected in $C_{f}$. By applying a pose-specific face detector on this much smaller region in the image, the cameras are able to quickly extract non-frontal face images and simultaneously index these faces into the corresponding face pose. Using this approach, it is possible to detect non-frontal faces at almost the same rate as for frontal faces. This system is easy to setup, does not require camera calibration and only depends on fundamental matrices of transformation between camera pairs.

- In order to evaluate the above face acquisition service in real time I have designed a portable embedded camera network testbed. This testbed consists a set of embedded cameras, and each camera assembled using Logitech pro 9000 web camera for imaging, 802.11 based wireless card for communication and ARM or Intel atom processor based embedded system for local processing. I have designed software that allows the cameras to automatically self configure in the network and to transmit data to base station for recognition and it also allows the remote programming of individual camera from the base station thus providing flexible and convenient framework. I have also integrated 
embedded camera with a create iRobot platform thus yielding mobile camera platform. In this thesis I have used this testbed in the context of face recognition but in general it can be used for any event surveillance applications. The testbed provides foundational infrastructure for camera networks based biometric and surveillance research and I also expect that this testbed will provide hands on experience for the students in the department.

\subsection{Thesis Outline}

The rest of this thesis is organized as follows. The chapter 2 discusses background information and related work. Chapter 3 describes opportunistic frontal face acquisition system and chapter 4 describes collaborative multi-view face acquisition system. The chapter 5 concludes this thesis and provides directions for the future work. 


\section{Chapter 2}

\section{Background information and Related work}

In the past decade, significant advances have taken place in the design of biometric systems that establish human identity based on physiological and behavioral characteristics such as face, iris, finger prints, voice, key stroke, gait and ear. In comparison with other modalities, human identification systems based on face and soft biometric features are more relevant in the context of unconstrained scenarios since they can be collected without knowledge or co-operation of a subject. This thesis primarily focuses on the use of a distributed camera network for human identification based on the face biometric, although methods developed in this thesis are also applicable for acquiring other soft biometric data and using these to support (and augment the accuracy of) identification based on the face biometric.

\subsection{Background information}

The operation of a facial recognition system can be broadly divided into two subsystems: 1. Face detection and 2. Face recognition.

- Face detection: A face detector is used to detect and extract face images present in an image. Face detection in an unconstrained environment considered as a challenging problem due to the following reasons:

Change of scale and pose: A face may appear in different poses because of in-plane 


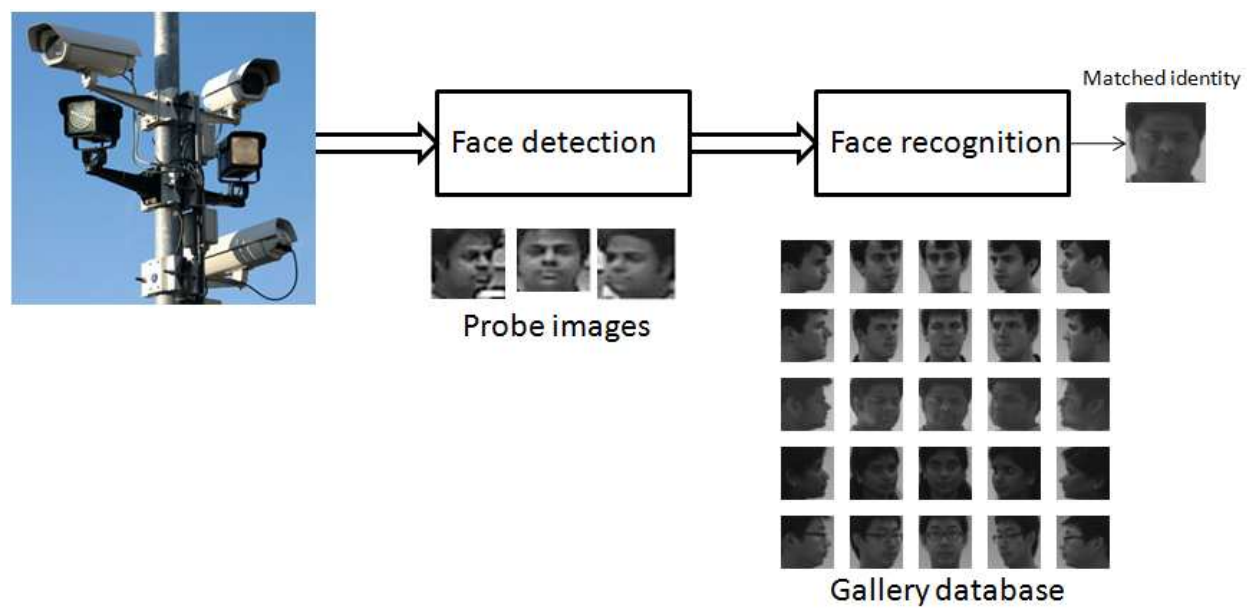

Figure 2.1: Face recognition system

and out of plane rotation w.r.t to the camera optical axis and the size of a face varies with user distance from the camera.

Expressions of face: Facial expressions such as laughing, talking cause significant variations in facial appearance.

Illumination and background complexity: Illumination variations and cluttered background environment also pose challenges to detection algorithms.

Occlusions: In real time, face images can be occluded by other people or objects in the scene.

- Face recognition: The face recognition subsystem first normalizes and aligns a probe face image, and then performs matching with the pre-acquired gallery database to determine the identity of the probe image. It establishes identity based on a similarity score between probe and gallery images. A face recognition algorithm must be robust to face variations due to pose, expression, resolution and resolution.

A distributed camera network can alleviate challenges posed by face detection in an unconstrained environment due to the redundancy offered by multiple views which can provide robustness against occlusions, variations in pose and illumination. However, such a multicamera setup also poses some challenges. The amount of data generated by multiple cameras can be too large to be transmitted and processed at a single location. If every frame captured 
by a camera is processed in a central location for face recognition, the system is unlikely to be capable of operating in real time. Therefore, local processing is needed prior to invoking the services of a fusion center. On the other hand, the local processing units are likely to be resource-constrained in order to be cost-efficient; further, too much of local processing will increase the overall recognition time. Hence, a balance between local processing capabilities and centralized fusion is needed. To achieve this balance, this work proposes the use of the multi-camera network to collaboratively acquire multi-view face images at a fast rate and the use of the central location (hereby referred to as base station) to perform matching of the opportunistically acquired probe images.

\subsubsection{Related work on multi-view face detection}

The goal of face detection is to determine whether a face exists in a given image or not, and to return the coordinates of the face if a face exists. In the last few years several face detection techniques have been proposed to detect faces in images. Image based face detection algorithms can be broadly classified into four categories [9]:

- Knowledge-based methods: These methods utilize a set of rules framed by the algorithm designer based on intuition of facial appearance to detect a face in an image. Kotropoulos and Pitas[10] et al. proposed a rule based face detection and it uses projection method to localize the facial features. Yang and Huang[11] et al. proposed hierarchical knowledge-based face detection method to localize face in an image and in this method set of rules are categorized into three levels to reduce the computational complexity. At the highest level general facial features such as uniform intensity regions and at the lowest level features such as eyes, nose and mouth regions are searched for candidate's face. These techniques are suitable for single face and simple background scenario.

- Feature invariant methods: These methods make use of facial features which are invariant to illumination and pose to localize the face in a given input image. In $[12,13]$ morphological and edge detection operations have been performed to extract facial features such as hair line, eyebrows, and mouth. The relation between extracted 
features studied to determine the existence of face. Even though these methods are robust to illumination they suffer from image noise and occlusion of facial features.

- Template matching methods: These methods utilize predefined face and facial feature templates for face detection. Sakai[14] used face contour and eyes template to model a face. In order to detect a face in an image, the image is scanned by a small window and correlation between the window region of an image and face contour template is computed to determine the regions which are likely to contain a face. Then eye and mouth templates are applied to detect a face. These methods are suitable for images which contain fixed size face images.

- Appearance-based methods: Appearance-based methods have shown superior performance over the above methods in terms of accuracy and computational speed. These techniques utilize machine learning algorithms and probabilistic models to build a classifier from a training dataset. In [15][16][17] authors proposed artificial neural network architectures for frontal face detection. To detect a face, neural networks are trained with positive and negative images to adjust the weights of nodes. Osuna et. al developed an efficient support vector machines training technique(SVM) and successfully applied it to detect frontal faces in gray level images. Support vector machine is a linear classifier and in the training phase optimal hyperplane computed to separate the face and non-face classes. Yang [18] used Sparse Network of Winnows learning architecture to localize faces with different face expressions and under different illumination conditions. Their method has shown improved face detection rate over SVM and neural network techniques but suffers from more false positive rate. In [7] Viola and Jones proposed rapid object detection using haar-like features to detect faces in images. It has been considered as a state of art algorithm till today, since it can able to detect faces rapidly while maintaining the accuracy.

The above techniques are proposed to detect frontal face images, but in real time $75 \%$ of face images are reported to be non-frontal. In the next section we discuss about various approaches towards multi-view face detection. Multi-view face detection techniques are broadly classified into two categories: 


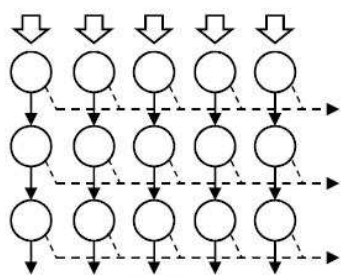

(a)

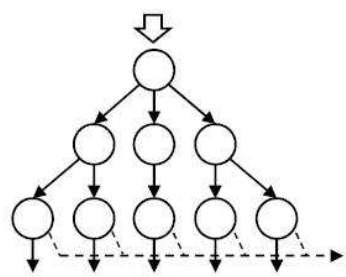

(c)

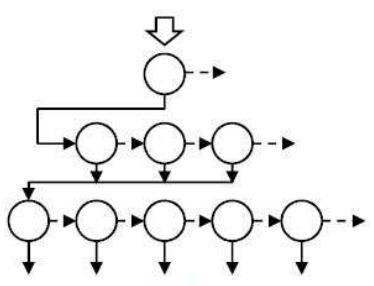

(b)

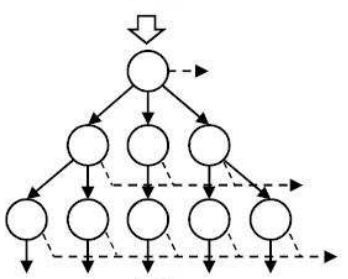

(d)

Figure 2.2: Classifier structures for multi-view face detection: Each circle indicates a classifier. The solid arrows are pass route, and the dashed arrows are reject route. (a) Parallel cascade, (b) Detector-pyramid, (c) Decision tree I, (d) Decision tree II

\section{Image based approaches}

Image based approaches are further classified into four categories based on classifiers arrangement in a detector:

- Parallel cascade structure: In [19] Huang et al. extended Viola and Jones frame work for multi-view face detection by training haar cascade classifier corresponding to each pose. In this method image sub window passes through all view based detectors as shown in figure. 2.2 and all classifiers results are combined to detect a face. This approach shows good performance but fails to perform in real time beacause all pose detectors are applied sequentially in order to search for a face in given sub window.

- Pyramid structure: In [20] Li proposed a pyramid structure to detect faces as shown in figure. 2.2. Their method uses hierarchical classifiers . First level is used to detect faces and non face patterns, second level to handle in-plane rotations $(-90$ to +90$)$ and the third level is used to detect specific pose of a face. This technique shows good performance over parallel cascade structure. 
- Decision tree structure - I: In [21] Viola and Jones extended their frame work in [7] for multi-view face detection. Their work uses decision tree structure - I as shown in figure. 2.2 to detect faces. The classifier in the early stages estimates the face pose and then applies pose specific classifier to detect a face in a sub-window. This method is robust and accurate but it results in false negatives since if the pose of a test image is misclassified whole detection process goes wrong.

- Decision tree structure - II: To overcome the problem in the above approach [21], Huang et al. [22] used vector boosting technique to build a decision tree classifier. In their approach each node computes determinative vector $\mathrm{G}(\mathrm{x})$ to determine sub branches in a next level, to pass test image for face detection. In this approach output of multiple branch classifiers are fused to take a decision instead of considering an output from a single branch.

\section{Video based approaches}

To improve detection speed associated with the image based approaches video based techniques have been proposed in $[23,24]$. In this approach, first face is detected and then it is tracked continuously in subsequent frames. These approaches work well for few subjects in a frame and tracking becomes complex when multiple people are present in a frame.

All of the above approaches use a video or image from a single camera for face detection and takes significant amount of time for detection. In contrast, the collaborative face acquisition framework proposed in this thesis exploits the multi-view geometry between cameras to acquire multi-view face images rapidly and accurately.

\subsection{Other related work}

Early surveillance systems $[25,26,27,28,29,30,31]$ are centralized, in which cameras acquire the data and send the whole data to a server for face detection and recognition. This approach takes significant amount of bandwidth which in turn increases network congestion and load on the server. Thus system performance deteriorates as the number of cameras 
increase in the network. In contrast, video analytic systems [32] are used for surveillance purposes and these systems employ a camera and dedicated hardware to acquire and perform face recognition task locally to overcome network problems. But these systems are incur heavy costs and do not collaborate with neighboring cameras to increase the performance of the system which is the focus of this thesis. In recent years, a number of different prototypical camera network systems have been developed to acquire face images in real time for recognition. However, face recognition imposes stringer requirements on the quality of acquired face images and therefore the use of a camera network for face recognition is much more challenging. To counter this challenge, many active vision based network systems have been proposed $[33,34,35]$ for face recognition. These systems use master-slave architecture in which combination of a fixed camera and a pan-tilt-zoom(PTZ) cameras are used to cover an assigned target area. In the above architecture fixed cameras continuously track the subjects and actively controls the PTZ camera to get a close up view of assigned subject. Each PTZ camera is typically assigned to acquire face images of a single subject at a time and configured continuously to cover multiple subjects in the scene. As a result, only short amount of time can be allocated to each subject and successful acquisition of suitable face images is contingent upon the subject retaining a suitable face pose while a camera is being configured. Also for reasons of scalability, typically a single camera is allocated per subject, thereby not utilizing the multiple views offered by the network to improve the chances of acquiring high quality face image. Instead of continuously tracking a subject at close quarters to eventually get a good view that is suitable for face recognition, the proposed approach relies on redundancy offered by multiple camera views to opportunistically acquire a suitable face image for identification.

Camera networks have been used for passive tracking of individuals to monitor their activities [25, 26, 27, 28]. Different techniques have been proposed to deal with overlapping field of views, handling occlusions and facilitating camera hand-offs in the context of tracking. There has also been research on deployment of camera networks to guarantee persistent surveillance as an object moves from one field of view to another. Above approaches utilize overlapping views to track the subject successfully but none of them used multi-view geometry to acquire face images. The proposed approach addresses the new frame work to 
collaboratively utilize the information between overlapping views in order to detect multi view face images. 


\section{Chapter 3}

\section{Opportunustic face acquisition system}

This section discusses the design of network infrastructure for face acquisition in real time and then describes software implementation for the same.

\subsection{System model}

The system that we consider in this project consists of a long linear network of embedded cameras deployed along both sides of a secured passageway such as an aisle or a corridor. Such a deployment models secured walkways at indoor public places. The embedded cameras are connected wireless to a central server either through one hop or multiple hops. As one or more human subjects walk through the network, cameras capture images of the subject under possibly different poses, resolution and even illumination. The requirement is for the subject to be recognized with high confidence using the series of images captured within the camera network. The system is subject to failures of individual units and occlusion effects. In this chapter, we specifically focus on the problem of distributed face recognition when a single person is within the camera network at any given time. Extending this to a multiperson recognition system is a subject of our future work. Note that the cameras that we place along the sides are passive cameras and do not track a subject upon detection. Instead we rely on opportunistically collected images from a dense set of cameras for identification. 


\subsubsection{Assembly of camera platform}

For object detection and recognition on an embedded smart camera system, algorithms have to be adapted and tailored to meet real-time constraints and environmental restrictions without significant loss in robustness and performance. The first challenge is the identification of an appropriate embedded platform. A number of different prototypical smart camera units have been developed $[36,37,38]$ in recent years. Fundamentally, these units possess an ARM or XScale class processor with a speed of around $400 \mathrm{MHz}$ along with a video capture unit. As a representative choice for these platforms, we evaluated the Intel imote2 platform for face recognition. While we were able to perform activities like edge detection on an image in the order of a few milliseconds, operations for face detection can be far more computationally intensive and required about 15-20 seconds on this platform. Moreover, the image quality obtained using the on-board multimedia unit was not sufficient for face recognition. In order to suit real-time face recognition, we assembled a smart camera platform using offthe-shelf components. The requirements include flexibility with respect to choice of cameras, networking capability, and availability of a DSP in case heavy computations are needed.

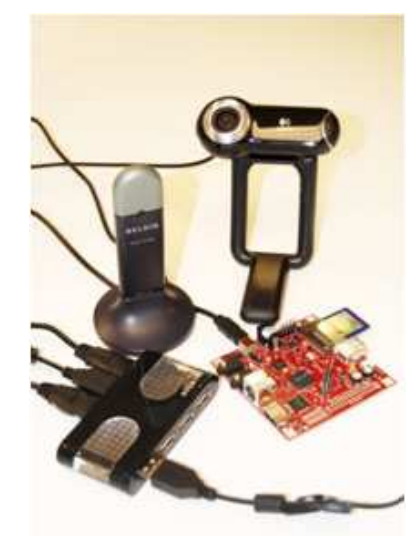

Figure 3.1: Smart camera unit: Assembled using a Beagleboard, Logitech 9000 camera and a 802.11 wireless card

For the experiments described in this paper, we use the OMAP3 processor based BeagleBoard [39] as our smart camera unit. The BeagleBoard is based on TI's OMAP3530 processor. Along with a $600 \mathrm{MHz}$ Cortex-A8 core, the OMAP3530 integrates TI's TMS320C64x core, a high-end DSP (digital signal processor) clocked at 430MHz. The processor supports 
Linux operating system and can be integrated with off-the-shelf USB enabled cameras generating medium or high resolution images. For our experiments we use the Logitech 9000 camera. While currently we use an 802.11 based wireless card, we can easily replace this with a low power wireless network using IEEE 802.15.4 enabled transceiver. Thus the assembled system provides us with flexibility with respect to camera as well as radio platforms. The assembled platform can be powered by a $5 \mathrm{~V}$ battery or external $\mathrm{AC}$ power.

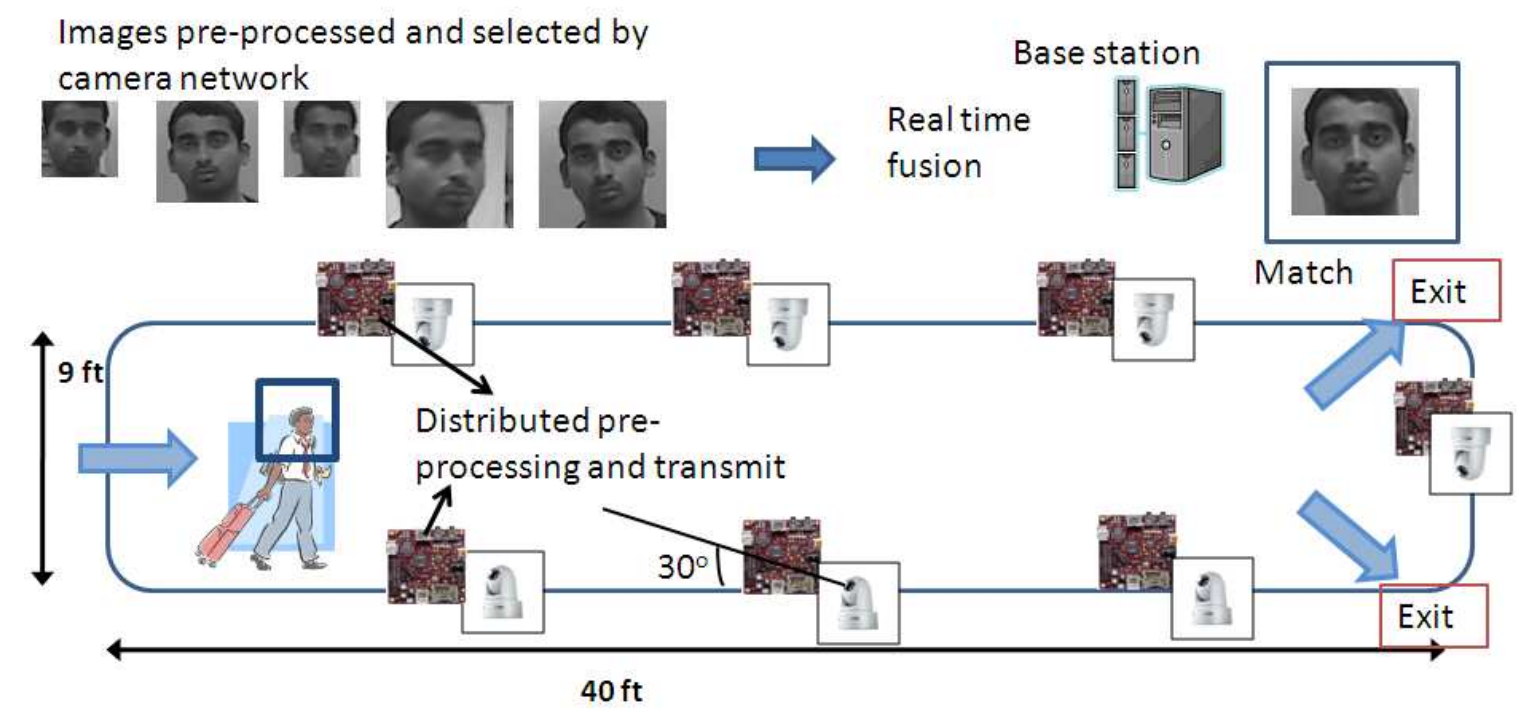

Figure 3.2: Schematics for network based face recognition

\subsubsection{Assembly of multi-camera network}

Next, we assemble a camera network using the above platform. Individual units are portable and self-configured into a programmable network that is attached to a centralized server. Figure 3.2 shows a schematic of the camera network for face recognition as described in this paper. Seven cameras are placed at a height of 6.5 feet from the ground with a 9 feet spacing long a length of 40 feet. In this work, we specifically consider the case where the cameras are placed at a height of 6.5 feet and are therefore able to acquire facial images that are frontal or side view. Using overhead or ceiling placed cameras for face recognition is beyond the scope of this work.

We do not use camera calibration information for face recognition. Therefore, the cameras 

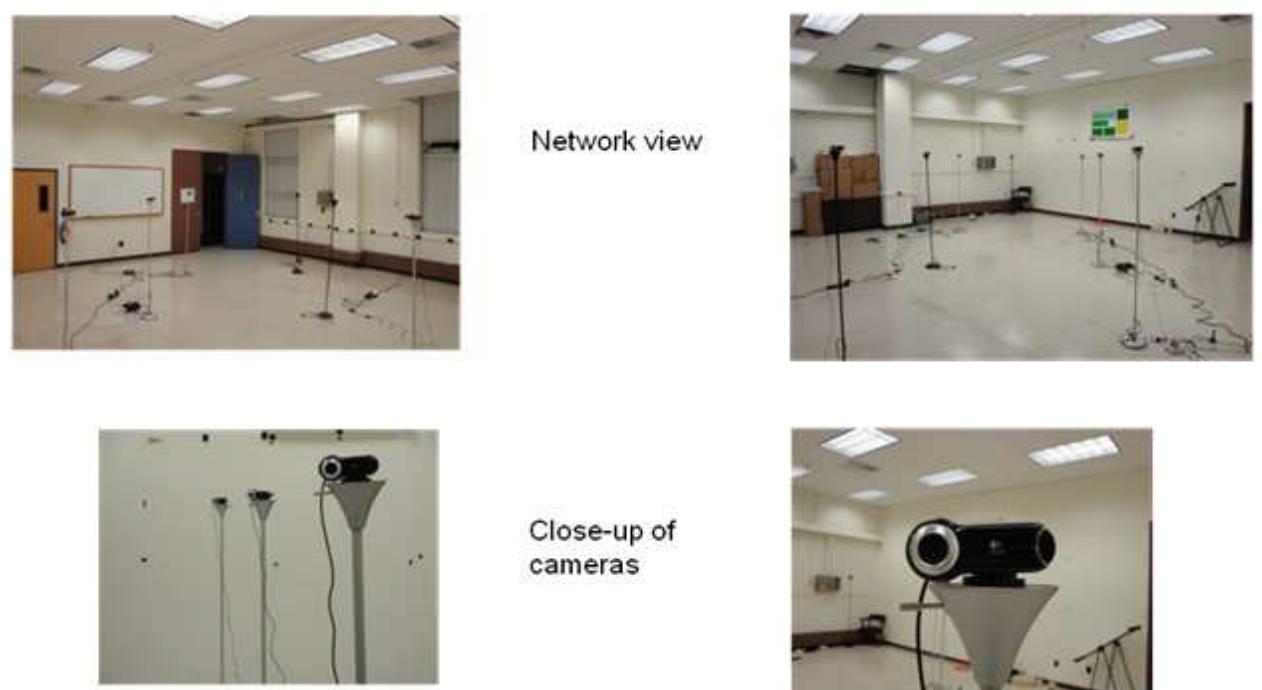

Network view

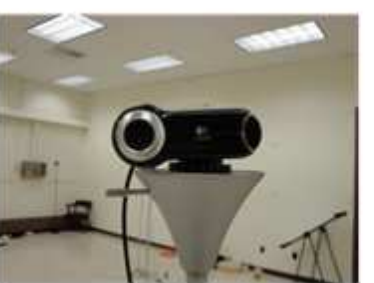

Figure 3.3: (Top) Deployment of smart camera network with 7 cameras for face recognition experiments. (Bottom) close-up view of individual camera; cameras deployed on 6.5 feet lamp posts

do not have to be tightly calibrated, thereby allowing approximate alignment and simplifying the deployment. The cameras are deployed facing the covered area but angled toward the direction of the entrance at angle of 30 degrees to be able to acquire frontal face images when the subject is staring at the direction of the exit. This is highlighted in Figure 3.2. The individual camera units can be programmed at run time. The cameras form a 1-hop network, but can be extended to form a multi-hop network. Figure 3.3 shows the deployment of our actual camera network for face recognition. The cost of an individual embedded camera unit along with accessories was $\$ 200$ and thus the total system cost for our 7 camera network is $\$ 1400$. We expect that with mass production of individual micro-controller boards, the cost will be significantly lower than this.

\subsubsection{Software implementation}

The software for face recognition is implemented over multiple devices: on the embedded devices and on the centralized PC. The objectives behind our distributed implementation are to reduce data transfer rates to the centralized processing unit and to exploit the redundancy offered by the network. On the embedded devices, we first implement an activity detector which is activated whenever a significant scene change is observed in consequent frames. 
This event triggers a face detector algorithm which determines if any frontal views of faces are present in the frame. A simple Haar cascade based face detector is implemented on the individual units. By computing only relative changes from a static background, the time required for face detection is significantly reduced. The captured images are scaled down to 320 x 240 pixels before transmitting them to the face detector module and this results in significant reduction in processing time while retaining the accuracy of face detection. We use an OpenCV based implementation of the Haar Cascade detector that is tuned to extract face segments that can be used for frontal face recognition. By virtue of the Haar Cascade detector (that is designed based on a set of training images), faces of small size, poor pose, illumination and resolution are filtered out. We specify a size of $22 \times 22$ as the minimum dimension for a detected face. The detected and extracted image sections are then transmitted to the central unit. This local processing serves to drastically cut down the data rate of transfer to the central unit. Moreover, by limiting the number of cropped images transmitted to the central unit, the computations that are to be performed by the central server are significantly reduced.

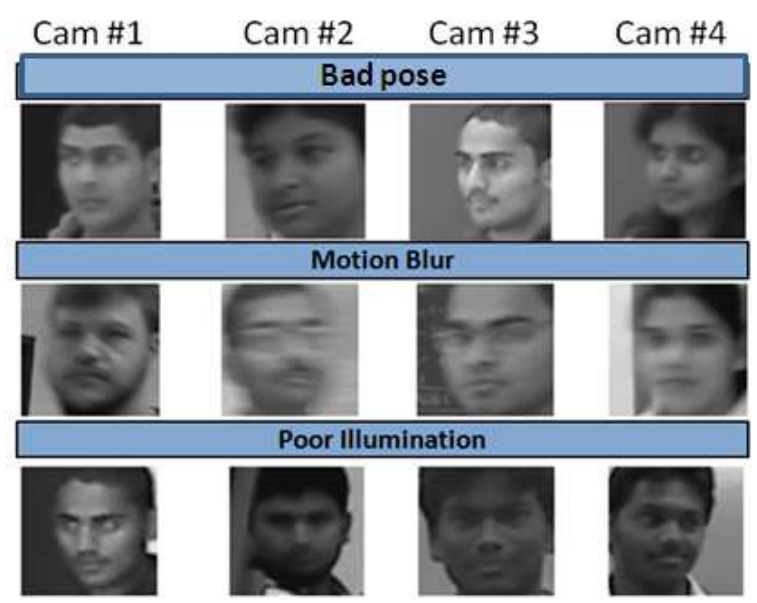

Figure 3.4: Images that did not qualify for transmission to the base station due to: Bad pose (top); motion blur (middle); poor illumination (bottom) 


\subsection{Performance evaluation}

\subsubsection{Experiment setup}

On the central unit, we use the redundant images provided by the embedded devices to perform face recognition. Commercial software such as Verilook or Identix G-6 can be used for this purpose. We use the L1 Identix software. Note that we have chosen to select only frontal face images for recognition. However, by expanding the training set for the Haar Cascade classifier to include side facial images, the filtering operation can be redefined. In order to evaluate the performance of the distributed face recognition system, we had 29 subjects walk through the network covering 40 feet in 9-10 seconds at a speed of about 2.75 mph. Some subjects were instructed to gaze at an arbitrary direction and walk whilst looking in that direction. This simulates the effect of people staring at something of interest along a secured passageway. Another set of subjects were instructed to move their faces arbitrarily even while walking. The camera network extracts filtered probe images and transmits them to the base station (i.e., the central PC). For each subject, 5 gallery images were recorded prior to the experiment under well illuminated and controlled settings. The probe images are not likely to be of the same quality. The subject gallery images are mixed with a set of 71 other subjects (5 images per subject) taken from the WVU Multi-biometric database. Each transmitted probe image is compared against each of the 500 gallery images and a match score is generated for each comparison. Let $p_{1}, p_{2}, \ldots, p_{n}$ be the $n$ probe images corresponding to a single subject walking through the passageway, and let $g_{1}^{i}, g_{2}^{i}, \ldots, g_{m}^{i}$ be the $m$ gallery images corresponding to identity $i$ in the gallery database. If $s_{k, l}^{i}$ is the match score generated by comparing $p_{k}$ with $g_{l}^{i}$, then the fused score corresponding to this identity is computed as,

$$
S_{p}^{i}=\max \left\{s_{k, l}\right\}_{k=1 \ldots n, l=1 \ldots m}
$$

If there are $N$ identities in the database, then the identity corresponding to the probe, $I(p)$, is computed as,

$$
I(p)=\arg \max _{i=1}^{N}\left\{S_{p}^{i}\right\}
$$


i.e., the gallery identity with the highest score is deemed to be the identity of the probe image.

During the process of filtering images prior to transmission to the base station, we apply a predetermined threshold on the resolution of a face image and the blur factor to discard frames. These thresholds are estimated based on an off-line analysis of the impact of different quality images on the match scores. If blurred and poor resolution images were to be transmitted to the base station, it will result in excessive processing time at the base station without adding value to the fusion. Fig. 3.4 shows example face images that were dropped due to poor pose, resolution and illumination respectively.

\subsubsection{Results}

Using the above experimental setup, we now describe both the network performance as well as the performance of face recognition in terms of accuracy and latency.

\section{Network performance}

Table 3.1 lists the time taken by individual camera units for various operations on the embedded camera. From the table we note the following. By using the background subtracted image for face detection, we cut down the processing time from 1.9 seconds to less than 500 ms. We save $90 \%$ of the bandwidth on each transmitted frame by extracting only the face portion of the image. Further, by transmitting only a small subset of frames we reduce the network level to a significantly low value thereby eliminating the probability of congestion at a central unit. By utilizing only the ARM processor, we are able to achieve about 1.5 frames per second when face detection is being performed and 8fps when no face recognition is performed. Transferring images from the ARM to the DSP requires significant memory transfer time and is therefore not deemed as practical for improving the speed of operations. However, if the cameras were directly interfaced to the DSP for image processing and only the network operations were handled by the ARM processor, the frame rates could be significantly enhanced. This requires porting of the face detector to the DSP and we are currently working on this implementation. 


\begin{tabular}{|c|c|}
\hline \hline Operation & Time \\
\hline \hline Camera Initialization & $100 \mathrm{~ms}$ \\
Frame capture time (960 by 720) & $35 \mathrm{~ms}$ \\
Background subtraction & $70 \mathrm{~ms}$ \\
face detection (entire image) & $1200 \mathrm{~ms}$ \\
Face detection (segmented image) & $470 \mathrm{~ms}$ \\
\hline
\end{tabular}

Table 3.1: Time taken for various processing operations on the assembled smart camera platform

\section{Frame rate vs Capture resolution}

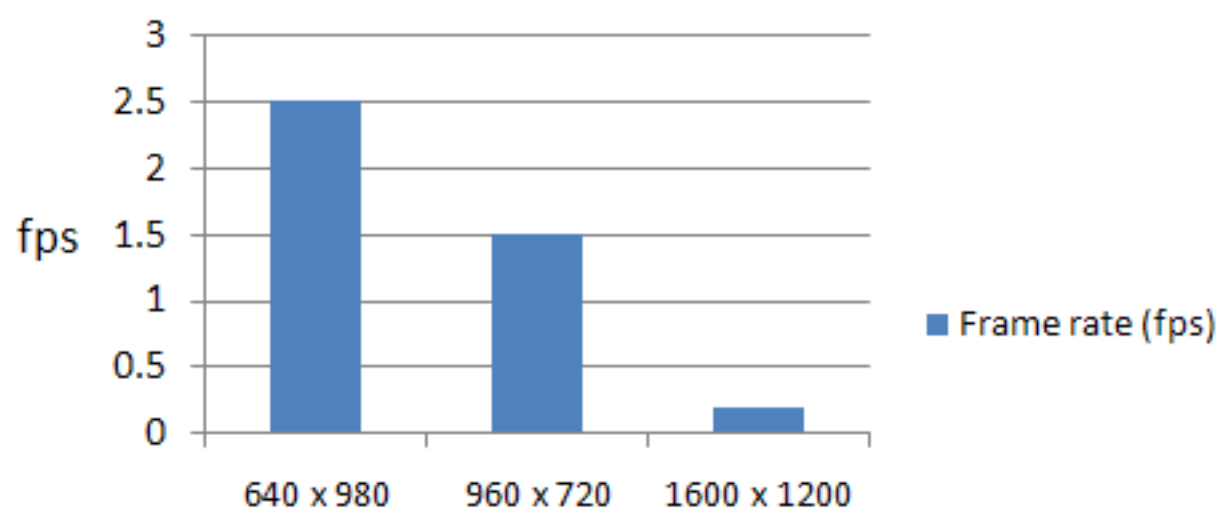

Capture resolution

Figure 3.5: Achieved Frame rate vs Capture resolution

Figure 3.5 shows the average frame rate at different face capture resolutions. Increasing resolution requires more processing time but is likely to yield better recognition accuracies. In our experiments, we choose a resolution of $960 \times 720$ and notice that it is sufficient for accurate face recognition under our current deployment scenario where cameras capture images at a distance of about 10 feet.

\section{Face recognition performance}

In Fig. 3.6 This section provides the details of the performance of the face recognition system as the number of cameras is varied. The figure shows the performance corresponding to rank 1, i.e., only when the top match from the gallery is considered. With all the seven 
cameras, we are able to achieve perfect recognition rate since all subjects are classified accurately. We then determine the accuracy when one camera fails. From our collected data we identify the camera that collected the most number of probes and in the figure we show the worst performance when that camera is assumed to have. We notice that the system is able to tolerate individual failures. We also see that when the system contains only one or two cameras, the recognition performance is rather poor.

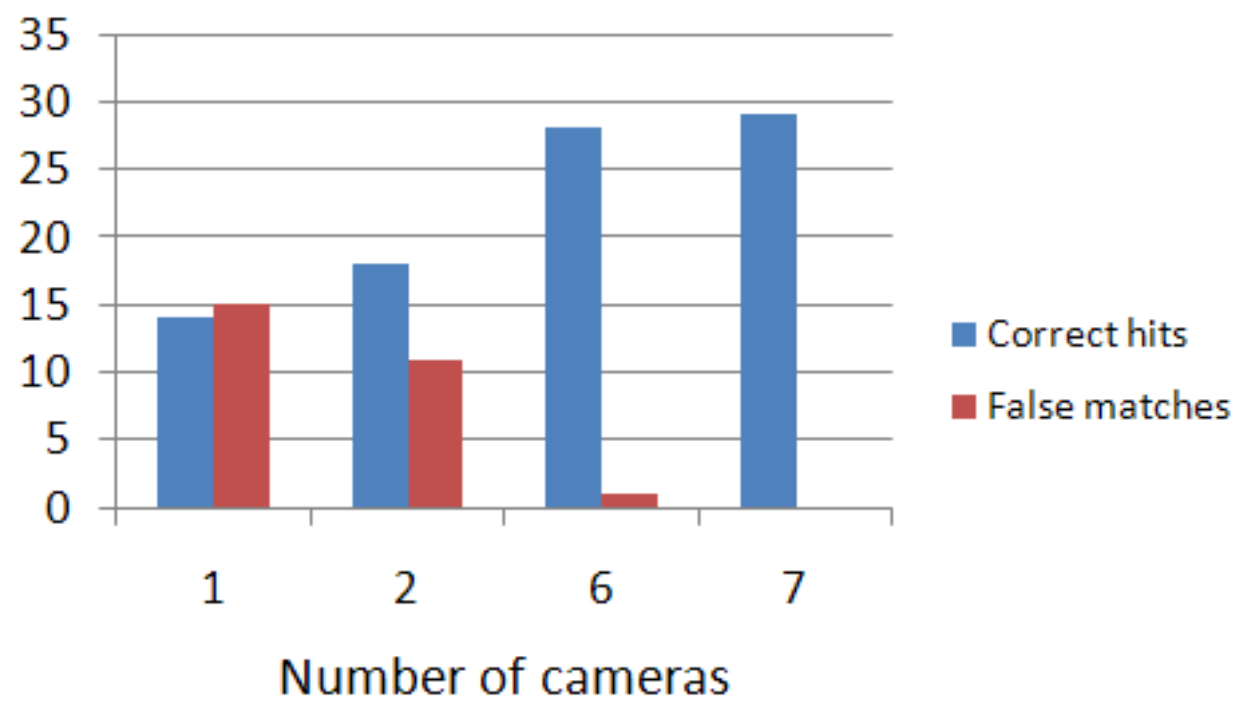

Figure 3.6: Correct recognition rate vs number of available cameras

In Figure 3.7, we show the ROC curves with different number of cameras in the system. We notice that we are able to achieve the good performance when all cameras are present and when one camera has failed.

Note that Fig. 3.7 represents a closed set analysis where the subject is always assumed to be present in the database and therefore a rank-1 evaluation suffices.

\section{Real time capability}

A single score generation on a $2.0 \mathrm{GHz}$ PC running face recognition software takes about 0.5 seconds. Thus by reducing the number of potential images transmitted to the base station, we are able to significantly reduce the processing time for face recognition. Now consider the case when the system is required to determine if the probe face is one among 


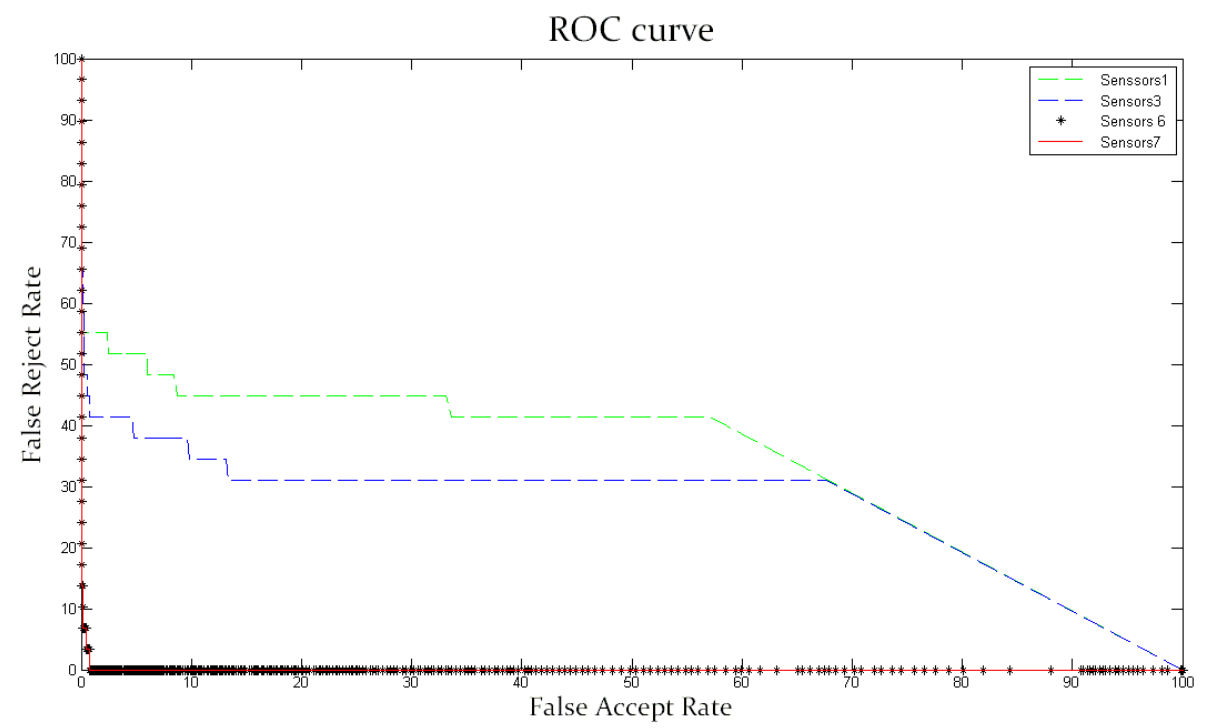

Figure 3.7: ROC curve of distributed face recognition system with different number of cameras activated

a small set of potential suspects. On average about 8 probe images are identified by our system per subject while passing through the network. Thus, if the number of gallery images is small then we can achieve face recognition in a few seconds after the subject has moved only a few meters. We note that if the cameras exchange quality information of the images collected and are able to identify the smallest subset likely to yield accurate face recognition, then the time required can be reduced even further. 


\section{Chapter 4}

\section{Collaborative multi-view face acquisition system}

In this chapter we discuss in detail about our network service for collaborative acquisition of multi-view face image. We describe system in two parts 1. System Model 2. system Operation

\subsection{System model}

Collaborative face acquisition system consists of a network of $N_{c}$ cameras with overlapping views that all are focused on a critical region such as entrances to public places and narrow corridors or walkways. The allowable distances between the cameras and the height of deployment will depend on the parameters of the cameras used for the system. For example if pan, tilt and zoom cameras are used, the cameras could be physically distant from the region and set to focus on the critical region. If fixed focal length, and low resolution cameras such as Logitech web cameras are used, they will have to be closer together. However, we do require that the cameras are able to acquire facial images that are either frontal view or side view (but not top view). For our specific experimental setting, we use a network of 3 cameras located along an arc of radius 10 feet. The cameras are deployed on tripods at a height of 7 feet from the ground with their principal axes parallel to the horizontal plane 


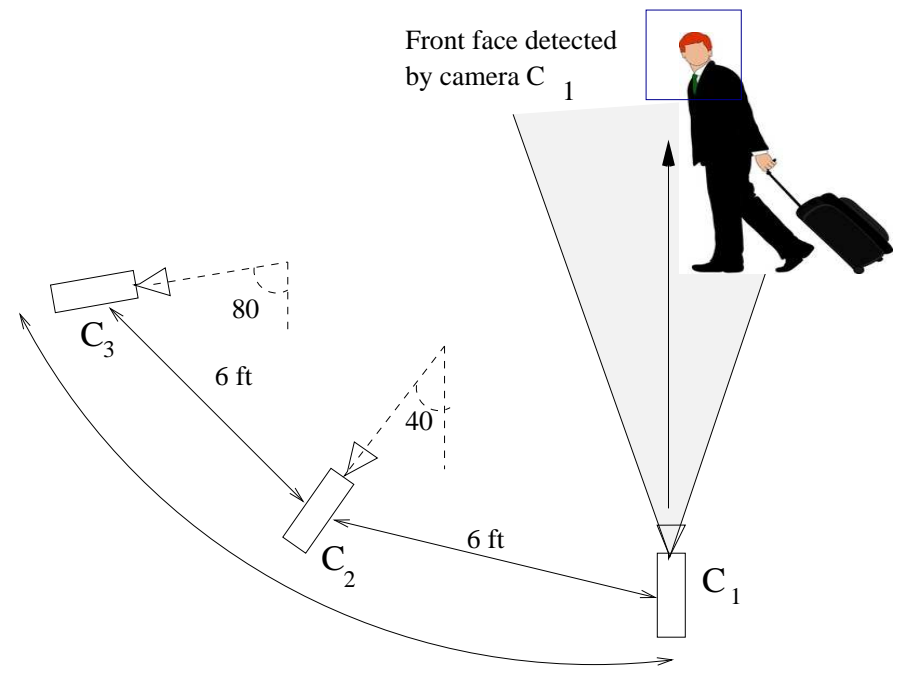

Figure 4.1: Our experimental deployment of 3 cameras. The cameras are deployed along an arc of radius 10 feet with a separation of 6 feet between the cameras along the arc as shown. The angles made by the principal axes of cameras $C_{2}$ and $C_{3}$ with that of camera $C_{1}$ are $40^{\circ}$ and $80^{\circ}$ respectively. The cameras are deployed on tripods at a height of 7 feet from the ground. All cameras run a frontal face detector. When a frontal face is detected on any camera, a notification is broadcast to other cameras.

and with a separation of 6 feet between the cameras along the arc as shown in Fig. 4.1. The angles made by the principal axes of cameras $C_{2}$ and $C_{3}$ with that of camera $C_{1}$ are $40^{\circ}$ and $80^{\circ}$ respectively. We arrange cameras in a specific configuration, which provides maximum variation between face poses so relative orientations between the cameras (the angles between principal axes of each pair of cameras) are assumed to be known. We assume that a clock synchronization algorithm is running on the nodes but we note that the clocks of any two nodes may not be in perfect synchronization at any time instant. Let $t_{s}$ denote the maximum clock synchronization error between any pair of cameras in milliseconds. This implies that the local clocks of any two cameras in network can be out of synchronization at most $t_{s}$ apart. The cameras are connected wirelessly to a fusion center where the transmitted face images are collected and may be used for face recognition. Our goal is to acquire face images corresponding to the following poses: frontal, left (or right) profile, partial left (or partial right) profile using camera network to improve the overall performance rate of surveillance system. We use the yaw angle (that measures the rotation of a face image along the vertical axis) to define front, profile and partial profile faces (illustrated in Fig. 4.2). We 


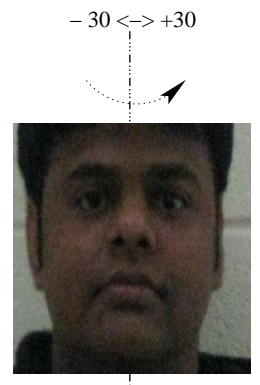

Front Face

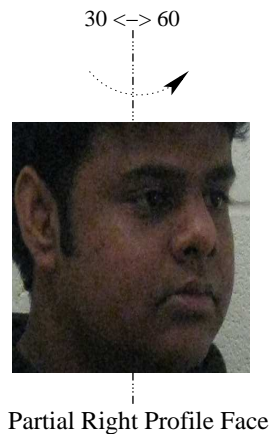

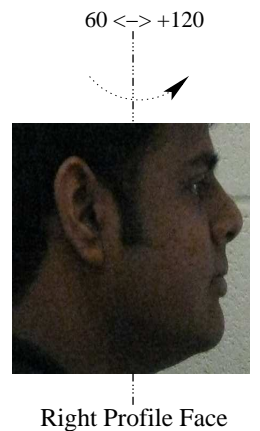

Figure 4.2: We classify faces into front, profile and partial profile based on the yaw angles

define a face image of a subject acquired by a camera to be frontal if the yaw angle made by the subject's pose ranges from $-30^{\circ}$ to $30^{\circ}$. We define a face image of a subject acquired by a camera to be partial left (partial right) profile if the yaw angle made by the the subject's pose ranges from $-30^{\circ}$ to $-60^{\circ}\left(30^{\circ}\right.$ to $\left.60^{\circ}\right)$. We define a face image of a subject acquired by a camera to be left (right) profile if the yaw angle made by the subject's pose ranges from $-60^{\circ}$ to $-120^{\circ}\left(60^{\circ}\right.$ to $\left.120^{\circ}\right)$. We have used the term side face to denote any non-frontal pose of the face.

\subsubsection{Epipolar geometry}

When two cameras observe a same point $\mathrm{X}(\mathrm{x}, \mathrm{y}, \mathrm{z})$ in $3 \mathrm{D}$ space, then the corresponding imaged points in two camera views are projectively equivalent and denoted as $x, x^{\prime}$. Epipolar geometry is used to describe projective mapping between the points $x, x^{\prime}$, which is independent of scene structure, camera internal parameters and relative pose. Epipolar geometry reduces corresponding point search space from $2 \mathrm{D}$ image to $1 \mathrm{D}$ epipolar line since point $x$ in one camera is constrained to lie on a epipolar line $l^{\prime}$ in the other image. Fundamental matrix is used to compute projective mapping between uncalibrated views and it is an algebraic representation of a epipolar geometry [40]. Properties of the Fundamental matrix given as:

- Epipolar constraint between corresponding points of two images of given as

$$
x^{\prime T} F x=0
$$




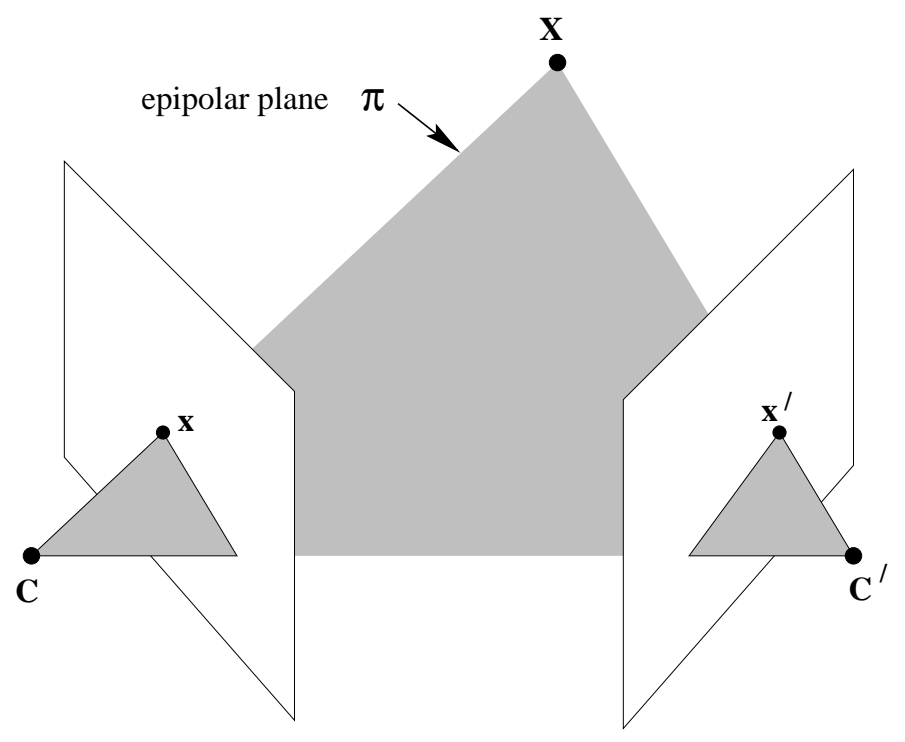

Figure 4.3: Point correspondence: The two cameras are indicated by their centers $C$ and $C^{\prime}$ and image planes. The camera centers, 3 -D space point $X$, and its image points $x$ and $x^{\prime}$ lie in a common plane

- For any point in one view, the corresponding epipolar line in another view given as

$$
l^{\prime}=F x
$$

- The relation between epipole and fundamental matrix given as

$$
F e=0
$$

\section{Fundamental matrix computation:}

Fundamental matrix is a $3 \times 3$ matrix of rank 2 and its computed based on corresponding image points between images and independent of camera calibration and camera internal parameters. Several techniques have been proposed to compute the F, but normalized-8 point algorithm has shown superior performance since input data normalized before solving linear equations for $\mathrm{F}$.

- Compute the feature points in an image using scale invariant feature transform[41], because common feature points in both images can be used to find the projective transformation between two views. 
- Use RANSAC algorithm to find the inliers, here inliers implies corresponding feature points in both images. Use 8-point algorithm method a fitting function for RANSAC to find matching feature points.

- Use normalized 8-point algorithm to find the fundamental matrix transformation between two views for a given set of matching features in two images.

\subsection{System operation}

In this section we discuss in detail about system that we use for multi-view face detection in real time. In camera network each embedded camera performs same operation and the operations performed on the embedded cameras are classified into the following four threads. The implementation has been illustrated with a pseudo-code in Fig. 4.4.

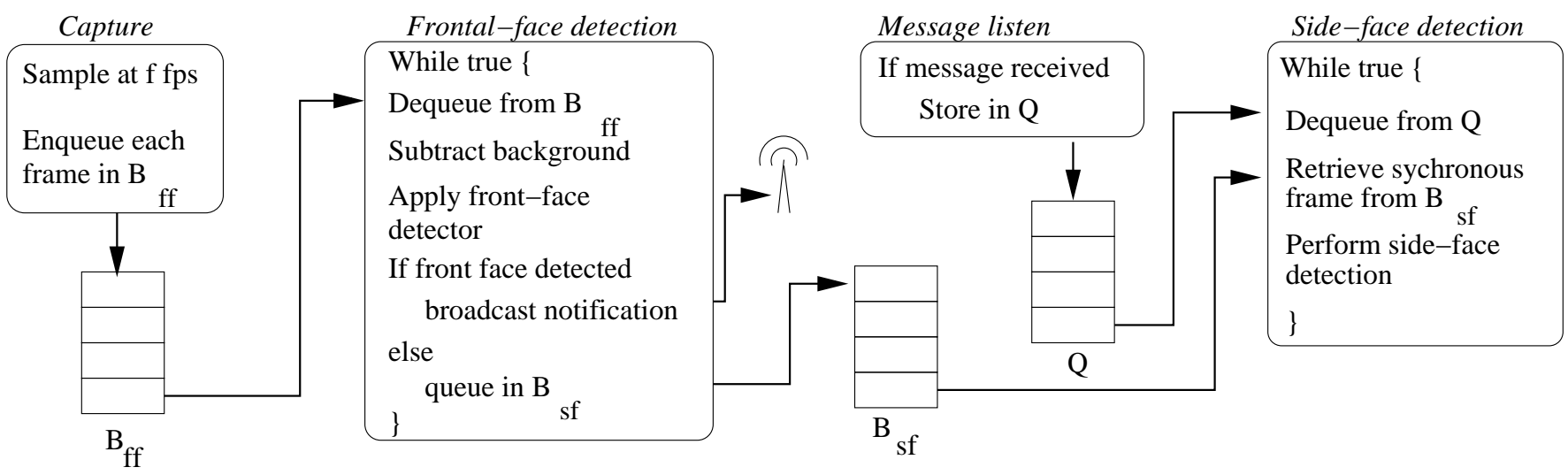

Figure 4.4: Pseudo-code for operations on each embedded camera. each node executes 4 threads: capture, frontal face detection, message listening and side-face detection. The capture thread samples images at $F$ fps and queues them in $B_{f f}$. The frontal face detection thread dequeues frames from $B_{f f}$ and applies frontal face detector on background subtracted images. If a face is detected, a notification is broadcast to other cameras, otherwise the background subtracted frame is stored in $B_{s f}$. The message listening thread queues any incoming message into $Q$. The side-face detection thread dequeues messages from $Q$, retrieves the synchronous frame corresponding to the message from $B_{s f}$ and performs the side-face detection procedure. 


\subsubsection{Capture}

The capture thread acquires images of the scene at $F$ fps and queues them into $B_{f f}$ buffer. Time taken by the camera to capture a frame can be given as $t_{f}=\frac{1}{F}$. We set the individual cameras to capture images whenever the local clock value is a multiple of $t_{f}$, and if there is any delay in capturing due to camera hardware then we encode timestamp value to closet multiple of $t_{f}$ from the time of capture. The timestamp of frame $x$ is defined as the time of capture of frame $x$ and denoted as $t(x)$. Also, we call frames $x$ and $y$ captured in two different cameras to be synchronous if $t(x)=t(y)$. Each image acquired by the capture thread is stored in buffer $B_{f f}$ along with its timestamp. Let $\left|B_{f f}\right|$ denote the maximum number of such images that can be stored in buffer $B_{f f}$. Also, recall that there could be a maximum clock synchronization error of $t_{s}$ time units between cameras. Due to both these reasons, images acquired by different cameras in the network with the same timestamp may not correspond to the same global time.

\subsubsection{Frontal face detection}

The frontal face detection thread dequeues the oldest frame from $B_{f f}$ to detect if a frontal face exists in the image. To detect frontal faces, it first perform background subtraction on an acquired frame. We consider initial set of frames to model the background based on median filtering and apply a threshold based differencing with respect to this image. Next, we apply morphological filters such as dilation and erosion to remove noisy pixels in background subtracted image and we use connected components labeling to find blob regions, where a face image could be present. We then apply an OpenCV implementation of the Haar Cascade based face detector [7] in each of the estimated foreground blobs. If a frontal face has been detected in a frame $x$, a notification message $M(c(x), t(x), w(x))$ is broadcast to all other cameras in the system, specifying the frame time $t(x)$, the location of the center of the face $c(x)$ and the width $w(x)$ of the bounding square around the detected face. If a frontal face is not detected, the background subtracted image is stored in side face buffer $B_{s f}$. Let $\left|B_{s f}\right|$ denote the maximum number of such background subtracted images that can be stored in buffer $B_{s f}$. 


\subsubsection{Message listening}

Each camera runs a server thread to listen messages from neighboring cameras upon receiving a message $M(c(x), t(x), w(x))$ from another camera, the message listening thread simply queues the message in a buffer $Q$ with maximum number of elements denoted by $|Q|$.

\subsubsection{Side face detection}

The side face detection thread retrieves messages from $Q$ one at a time for side face detection. The timestamp $t(x)$ in the message $M$ is used to retrieve the frame $y$ in the buffer $B_{s f}$ whose timestamp $t(y)$ is equal to the time $t(x)$ corresponding to frame $x$. Frame $y$ is then removed from the buffer $B_{s f}$ and a side-face detection procedure is run on frame $y$.

We use epipolar geometry to detect the side face in the frame $y$ : if two cameras $C_{1}$ and $C_{2}$ observe the same scene point $W(X, Y, Z)$ and if the image point corresponding to $W$ in $C_{1}$ is $P_{1}(x, y)$, then the image point $P_{2}(x, y)$ corresponding to $W$ in $C_{2}$ must lie on the epipolar line corresponding to $P_{1}(x, y)$ [42]. The fundamental matrix is an algebraic representation of this epipolar geometry. Given the fundamental matrix $F_{12}$ between cameras $C_{1}$ and $C_{2}$, the relation between $P_{1}$ and $P_{2}$ is given by the following equation:

$$
P_{2}^{\prime} F_{12} P_{1}=0
$$

The epipolar line corresponding to point $P_{1}$ in camera $C_{2}$ is described by:

$$
l_{2}=F_{12} P_{1}
$$

For our experimental setting, we compute the fundamental matrices between each pair of cameras off-line by using SIFT features for finding corresponding points, estimating the matrices using the normalized 8-point algorithm and then using RANSAC [43] to remove outliers from the detected keypoints [42]. We provide the fundamental matrices to the cameras before the experiments. Using the fundamental matrix $F_{r s}$ we project the point $c(x)$ (the centroid of the frontal face detected) to a corresponding epipolar line in the synchronous frame $y$ of the camera receiving the message (Fig. 4.5). We then determine the segment of the epipolar line that intersects with the background subtracted image retrieved from $B_{s f}$ 

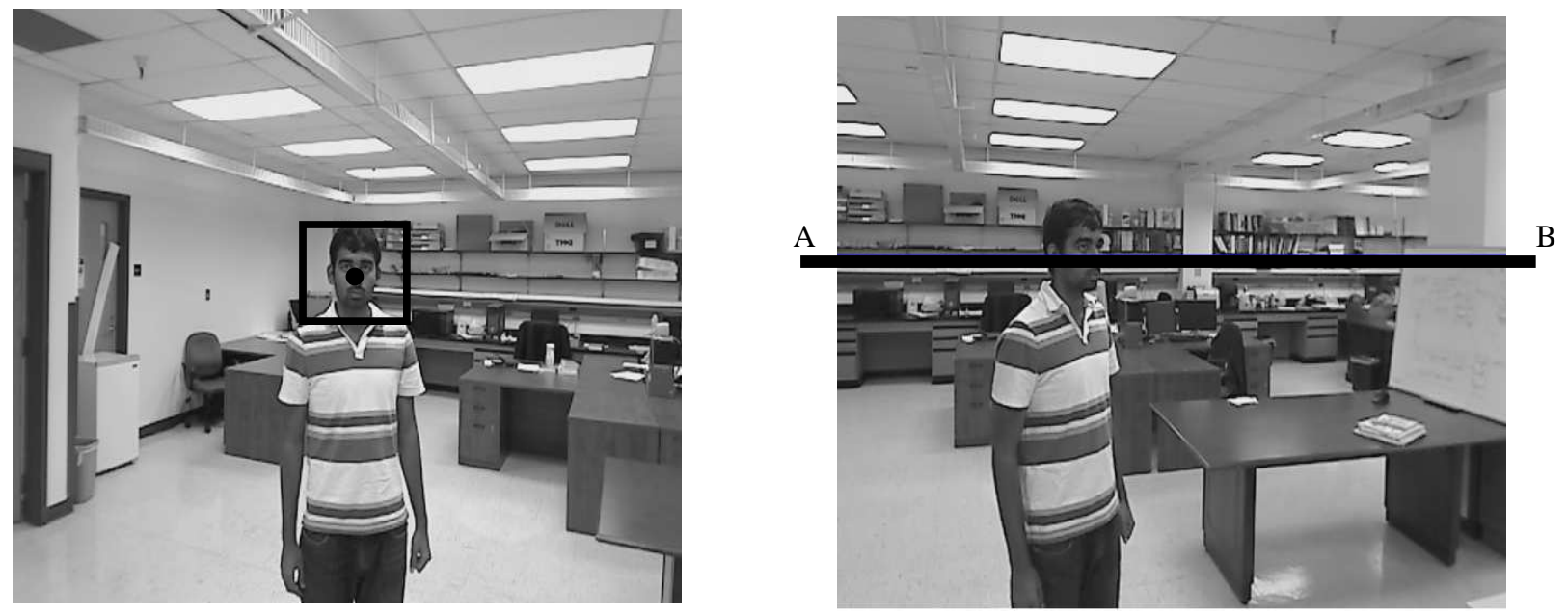

Figure 4.5: Using camera network geometry for side face detection. When a frontal face is detected on any camera, a notification is broadcast to other cameras specifying the center of the detected face. Epipolar geometry is used to project this point to a corresponding epipolar line (shown as $A B$ ) in the other cameras. The other cameras apply a pose-specific side-face detector in a small region surrounding the segment of the epipolar line that intersects with the background subtracted image.

and extract a square block of size $W \times W$ pixels around the center of this segment, where $W$ is set to be equal to the width of the detected frontal face image. Based on the relative camera orientations, we determine the expected pose of a side face and apply the side-face detector corresponding to the particular pose class on the extracted square block. For our experiments, we have trained face detectors for the left partial profile and left profile faces using an OpenCV implementation of the method described in [8]. To detect right partial profile and right profile faces, we apply the same detectors on the mirror images of the block.

\subsubsection{Buffer management}

Let $t_{n d}$ denote the maximum network delay incurred between transmission and reception of a notification message. Let $t_{f f}$ and $t_{s f}$ denote the processing times for frontal face detection and side face detection respectively. In this section, we analyze requirements on $\left|B_{s f}\right|$ to ensure that synchronous frames always exist in the respective buffer $B_{s f}$ of a camera when a notification message is being processed. We also analyze impact of $t_{n d}, t_{s}, t_{f f}, t_{s f}$ and $t_{f}$ on the expected number of frontal face and side face images that can be processed. For ease of presentation, we divide our analysis into the following cases. 
R1: $t_{n d}=0, t_{s}=0, t_{s f}+t_{f f}<t_{f}$ In this case both the side-face and frontal-face processing can be finished before a new frame is sampled. Moreover the network delay is 0. Hence we only need to set $\left|B_{f f}\right|=1,\left|B_{s f}\right|=1$ and $|Q|=1$. The expected number of frontal $\left(N_{f f}\right)$ and non-frontal $\left(N_{s f}\right)$ faces that can be detected by a camera over a time $T$ is given by the following equations.

$$
\begin{aligned}
& N_{f f}^{R 1}=\frac{T}{t_{f}} \\
& N_{s f}^{R 1}=\frac{T}{t_{f}}
\end{aligned}
$$

R2: $t_{n d}>0, t_{s}>0, t_{s f}+t_{f f}<t_{f}$ In this case also, a camera can finish both frontal and side-face processing before a new frame is sampled. But since $t_{n d}>0$, any message retrieved from $Q$ will have a timestamp that is old by at most $t_{n d}+t_{f f}$ (since it takes at most $t_{f f}$ time for frontal face processing). Moreover, if $t_{s}>0$ and the camera detecting the frontal face lags behind any side face processing camera by $t_{s}$ units, then the message retrieved from $Q$ could have a timestamp that is old by at most $t_{n d}+t_{f f}+t_{s}$. Therefore, in order to be able to retrieve a frame from $B_{s f}$ corresponding to the timestamp of the incoming message, the condition on $\left|B_{s f}\right|$ is given by the following equation.

$$
\left|B_{s f}\right|>\frac{t_{n d}+t_{f f}+t_{s}}{t_{f}}
$$

Note that if the camera detecting the frontal face is ahead of the other cameras by $t_{s}$ units and if $t_{s}>t_{n d}+t_{f f}$, then the frame corresponding to the timestamp in the incoming message will not be found in the side face processing cameras. The clocks in the side-face processing cameras in this case have not reached the clock value in the frontal face processing camera. In this case, the image with the most recent timestamp in $B_{s f}$ is used for performing side-face detection. Thus we see that the clock synchronization error between cameras causes the processing of side-faces to occur on frames that are apart by at most $t_{s}$ time units.

The expected number of frontal $\left(N_{f f}\right)$ and non-frontal $\left(N_{s f}\right)$ faces that can be detected by a camera over a time $T$ remain unchanged from case $R 1$ and are given by Eq. 4.6 and Eq. 4.7 respectively. 
R3: $t_{n d}>0, t_{s}>0, t_{f f}>t_{f}$ In this case, the frontal face processing time is greater than $t_{f}$. The side-face processing time $t_{s f}$ can be greater or smaller than $t_{f}$. However, since each side-face processing camera also runs a frontal face detector, the average time to process each message from $Q$ is $t_{s f}+t_{f f}$ which is greater than $t_{f}$. Therefore, new frames will be captured into $B_{f f}$ and new messages can arrive in $Q$ before the earlier ones are processed. Now, if we assume that the system will always remain active (in other words, there will be a human subject in the scene at all times), then even if we buffer frames in $B_{f f}$ and $Q$, there will be no $i d l e$ time to process these frames. We can therefore set $\left|B_{f f}\right|=1$, and $|Q|=1$. Thus the frames cannot be processed at the sampling rate and only the most recently acquired frame and most recently received message are queued in $B_{f f}$ and $Q$ respectively.

We now determine the size of $B_{s f}$ required so that for any frame in which a frontal face has been detected, we can find the corresponding synchronous frame in $B_{s f}$. We note that $|Q|=1$ and the maximum time before which this message is retrieved by a camera for detecting non-frontal faces is bounded by $t_{f f}+t_{s f}$. Now from the discussion in case $R 2$, we note that the timestamp in the message that is retrieved is old by $t_{n d}+t_{f f}+t_{s}$. So, the required size of buffer $B_{s f}$ is determined by the following equation.

$$
\left|B_{s f}\right|>\frac{2 t_{f f}+t_{s f}+t_{n d}}{t_{f}}
$$

The expected number of frontal $\left(N_{f f}\right)$ and non-frontal $\left(N_{s f}\right)$ faces that can be detected by a camera over a time $T$ are given by the following equations.

$$
\begin{gathered}
N_{f f}^{R 3}=\frac{T}{t_{f f}} \\
N_{s f}^{R 3}=\frac{T}{t_{f f}+t_{s f}}
\end{gathered}
$$




\subsection{Performance evaluation}

\subsubsection{Experimental setup}

In order to evaluate the performance of our data acquisition system, we implement it on a 3 node embedded camera network (schematics shown in Fig. 4.1). We assemble an embedded camera using a Logitech 9000 camera, a $1.6 \mathrm{GHz}$ Intel Atom 230 processor based motherboard from Acer [] and an IEEE 802.11 based wireless card. We consider one human subject in the scene at a time. Each subject stands at a distance of approximately 10 feet from the cameras (close to the center of the arc) facing any one of the 3 cameras. Note that, if the subject is facing camera $C_{1}$ as shown in Fig. 4.1, then the pose estimated by camera $C_{2}$ and $C_{3}$ are right partial profile and right profile respectively. We have tested the system with 10 different subjects with approximately 15 minutes of data collected for each subject.

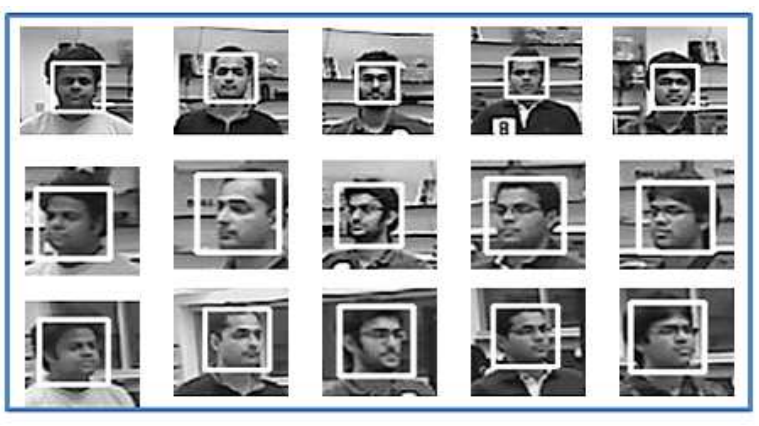

(a)

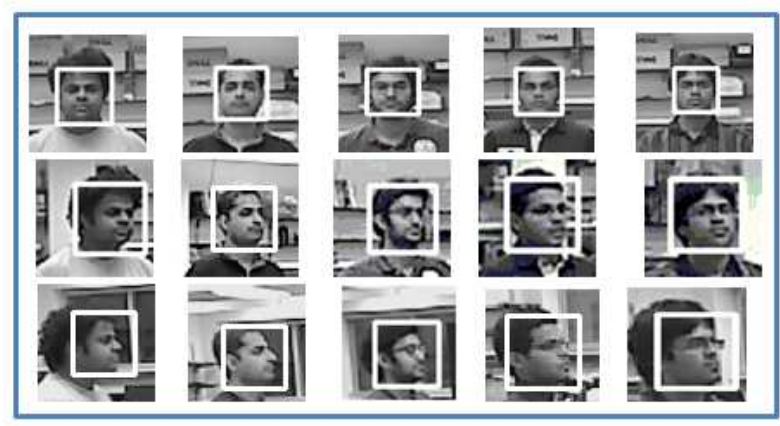

(b)

Figure 4.6: Example face images detected by our acquisition service. The white rectangles indicate the box enclosing the detected faces in each pose. Face images in each column are extracted from synchronous frames in the three cameras. (a) Images acquired with subjects facing $C_{2}$ : (Top) Frontal face (Middle) Left partial profile face (Bottom) Right partial profile face. (b) Images acquired with subjects facing $C_{1}$ : (Top) Frontal face (Middle) Right partial profile face (Bottom) Right profile face.

We use the Network Time Protocol (NTP) [44] for achieving clock synchronization between nodes and empirically observe a synchronization error of $<12 \mathrm{~ms}$. Alternatively we could also use a completely decentralized clock synchronization protocol and recent papers have demonstrated sub-millisecond accuracies [45] with extremely small communication costs and synchronization messages sent only once every 10 seconds. The impact of the clock synchronization error is that images grabbed at two cameras with the same timestamp may 
not correspond to the same global time. However, we note that with an error of even a few milli-seconds, a subject could not have moved much in that time.

\subsubsection{Results}

We perform experiments in two environments: one with a lot of clutter in the background (this environment is shown in Fig. 4.5) and the other one with a relatively plain background. Images are sampled by each camera at 25 fps. Thus $t_{f}=40 \mathrm{~ms}$. In Table 4.1 , we show the average execution times for the different processing modules in our system. In Table 4.2, we show the number of frames that are processed per second for detecting frontal faces and side faces. The frontal face detector is applied on background subtracted regions and sometimes applied even on spurious blobs detected as the foreground. The side-face detector on the other hand is applied only on a much smaller region that is corroborated by the frontal face detecting camera. We note from Table 4.2 that the number of frames processed per second for detecting frontal and side faces conform to the rates shown in Eq. 4.10 and Eq. 4.11 respectively when accounting for the operating system overhead. For instance in a clear background we observe that the average value of $t_{f f}=81 \mathrm{~ms}$ and average value of $t_{s f}=15$ ms and accordingly 11 frames can be processed per second for detecting frontal faces and 10 frames can be processed per second for detecting side faces in a clear background.

\begin{tabular}{||c|c|c||}
\hline \hline Operation & $\begin{array}{c}\text { Time }(\mathrm{ms}) \\
(\mathrm{clear})\end{array}$ & $\begin{array}{c}\text { Time }(\mathrm{ms}) \\
(\text { cluttered })\end{array}$ \\
\hline \hline Image capture and storage & 2 & 2 \\
\hline Background subtraction & 2 & 3 \\
\hline Dilation & 2 & 2 \\
\hline Frontal face detection & 75 & 102 \\
\hline \hline Total $t_{f f}$ & 81 & 109 \\
\hline \hline Total $t_{s f}$ & 15 & 15 \\
\hline \hline
\end{tabular}

Table 4.1: Processing times: Multi-view face detection in clear and cluttered background

The actual number of frontal and side faces detected correspond to the output of the detector itself. The difference between frames processed and faces detected gives a measure of the false negatives for the respective detectors. In a clear background, the number of frontal faces detected per second are almost equal to the number of frames processed per 
second. All the frontal faces detected are notified to the other cameras and the number of side faces detected per second in each camera matches the frontal face detection rate. In a cluttered background, the number of missed detections for frontal faces are high and yields a frontal face detection rate of 6 faces per second and as seen in Table 4.2, the side face detecting cameras are able to match this detection rate.

\begin{tabular}{||c|c|c||}
\hline \hline Rates per second & Clear & Cluttered \\
\hline \hline Frontal face processed & 11.1 & 8 \\
\hline Frontal face detected & 10.2 & 6.05 \\
\hline Side-face processed & 10 & 5.5 \\
\hline Side-face detected & 9.7 & 5.2 \\
\hline \hline
\end{tabular}

Table 4.2: Detection rates for frontal and side faces

The number of falsely detected side-faces using our acquisition service were negligible (close to $1 \%$ of the total number of side faces detected in our experiments). By selectively applying the side-face detector on regions corroborated by the frontal face detecting camera, we are able to achieve this low false alarm rate. The maximum network delay is observed to be $50 \mathrm{~ms}$, but we note that this only affects the size of $B_{s f}$ and not the overall face detection rate. We also note that the required buffering is very low (approximately 10 frames). By transmitting only the face images, that are on average $60 \times 60$ pixels in size, we are able to reduce communication bandwidth by $98 \%$ compared with transmitting the entire image $(640 \times 480$ pixels $)$ and by $80 \%$ when compared with transmitting the background subtracted image $(100 \times 200$ pixels on average $)$. By performing face detection and simultaneously estimating the pose, we are also able to reduce significant processing time at the fusion center for face recognition. 


\section{Chapter 5}

\section{Conclusions and Future work}

\subsection{Conclusions}

This thesis presented the collaborative multi-view face acquisition service that can be used to support face recognition system. To evaluate the opportunistic frontal face detection system, we considered a system with a fixed set of cameras deployed along the sides of a corridor to acquire face images while people walking through the network in the corridor. Experiment was conducted with 29 subjects walking through the network in isolation covering a distance of 35 feet in 7 to 8 seconds. The redundant views offered by different cameras enable face acquisition even when subjects were walking arbitrarily through the network. Experimental results show that the system was able to tolerate individual camera failure and also reduce the bandwidth utilization by transmitting face images only. We also note the limitations of our current approach. Currently, we utilize only the ARM processor on the Beagleboard which results in low frame rates. As a result, the system is likely to yield good performance only when the subjects move slowly across the network. The above problem can be alleviated by utilizing Beagleboard DSP processor for image processing. The above system performance relies on frontal face acquired by the system. However, in unconstrained environments it is not always possible to get high quality face images, in which case multi pose faces images can be used to yield high recognition rates. To acquire multi pose images we proposed a new framework for multi-view face acquisition which utilizes multi-view geometry and inter-camera communication to decrease computation complexity. 
Experimental results show that multi pose face detection rate was approximately equal to the frontal face detection rate, and face acquisition service was light-weight in terms of processing complexity. It has low buffering requirements and is appropriate for implementing on resource constrained smart camera platforms [27]. It has to be noted that face detectors based on haar-like features are used in the experiments for face detection, instead they could be replaced with other pose-specific face detectors to enhance the detection speed. Buffer management scheme is proposed to maintain frame synchronization between cameras in order to overcome network and camera hardware delays. This approach can be efficiently utilized when cameras have an overlapping field-of-view and works for fixed cameras only.

\section{$5.2 \quad$ Future work}

As a next step, we would like to integrate multi-view face image acquisition framework with multi pose face recognition techniques [46] to quantify the performance of a face recognition system and would like to evaluate the system performance with multiple subjects in the network. There has been significant research in image and score level fusion[47, 48], but these techniques work well for off-line data and we would like to investigate fusion techniques in dynamic scenarios where data is continuously updated for recognition. 


\section{References}

[1] U. Park and A. K. Jain. Face matching and retrieval using soft biometrics. IEEE Transactions on Information Forensics and Security (TIFS), 5(3):406-415, 2010.

[2] I.A.Kakadiaris, H.Abdelmunim, W.Yang, and T.Theoharis. Profile-based face recognition. In 8th IEEE international Conference on Automatic Face and Gesture Recognition, pages $1-8,2008$.

[3] K.W Cheung, J. Chen, and Y.S. Moon. Pose-tolerant Non-frontal Face Recognition using EBGM. In 2nd IEEE International Conference on Biometrics: Theory, Applications and Systems, 2008.

[4] F.Yang, M.Paindavoine, H.Abdi, and D.Arnoult. Fast image mosaicing for panoramic face recognition. Journal of Multimedia, 1(2):14-20, 2006.

[5] B. Bhanu and X. Zhou. Face recognition from face profile using dynamic time warping. In International Conference on Pattern Recognition, volume 4, pages 499-502, 2004.

[6] C. Huang, H. Ai, Y. Li, and S. Lao. High-performance rotation invariant multiview face detection. IEEE Transactions on Pattern Analysis and Machine Intelligence, 29(4):671 -686, April 2007.

[7] P. Viola and M. Jones. Robust real-time face detection. International Journal of Computer Vision, 57:137-154, 2004.

[8] R. Lienhart and J. Maydt. An extended set of haar-like features for rapid object detection. In IEEE ICIP, 2002.

[9] M. Yang, David J. Kriegman, and N. Ahuja. Detecting faces in images: A survey. IEEE Transactions on Pattern Analysis and Machine Intelligence, 24:34-58, 2002.

[10] C. Kotropoulos and I. Pitas. Rule-based face detection in frontal views. Acoustics, Speech, and Signal Processing, IEEE International Conference on, 4:2537, 1997.

[11] Guangzheng Yang and Thomas S. Huang. Human face detection in a complex background. Pattern Recognition, 27:53-63, 1994.

[12] H. P. Graf, T. Chen, E. Petajan, and E. Cosatto. Locating faces and facial parts. In IEEE International Conference on Automatic Face and Gesture Recognition, 1995. 
[13] S. Sirohey, M. Begum, A. Sirohey, and Z. Sirohey. Human Face segmentation and identification. Technical Report CS-TR-3176, University of Maryland, 1993.

[14] T. Sakai, M. Nagao, and S. Fujibayashi. Line extraction and pattern detection in a photograph. Pattern Recognition, 1:233-248, 1969.

[15] H. Rowley, S. Baluja, and T. Kanade. Neural network-based face detection. In Proceedings of the IEEE Computer Society Conference on Computer Vision and Pattern Recognition, pages 203-208, 1996.

[16] T. Agui, Y. Kokubo, H. Nagashashi, and T. Nagao. Extraction of facerecognition from monochromatic photographs using neural networks. 1992.

[17] Christophe Garcia and Manolis Delakis. A neural architecture for fast and robust face detection. In International Conference on Pattern Recognition, pages 44-47, 2002.

[18] M. Yang, D. Roth, and N. Ahuja. A snow-based face detector. In Advances in Neural Information Processing Systems 12, pages 855-861. MIT Press, 2000.

[19] B. Wu, H. Ai, C. Huang, and S. Lao. Fast rotation invariant multi-view face detection based on real adaboost. In In Sixth IEEE International Conference on Automatic Face and Gesture Recognition, pages 79-84, 2004.

[20] S. Li, L. Zhu, Z. Zhang, A. Blake, H. Zhang, and H. Shum. Statistical learning of multi-view face detection. In European Conference on Computer Vision, pages 67-81, 2002.

[21] Michael J. Jones and Paul Viola. Fast multi-view face detection. In Computer Vision and Pattern Recognition.

[22] Chang Huang, Haizhou Ai, Yuan Li, and Shihong Lao. High-performance rotation invariant multiview face detection. IEEE Transactions on Pattern Analysis and Machine Intelligence, 29:671-686, 2007.

[23] J. Wu and M. Trivedi. An integrated two-stage framework for robust head pose estimation. In $A M F G^{\prime} 05$, pages 321-335, 2005.

[24] Z. Zhang, G. Potamianos, M. Liu, and T. Huang. Robust multi-view multi-camera face detection inside smart rooms using spatio-temporal dynamic programming. In $F G^{\prime} 06$, pages 407-412, 2006.

[25] Y. Yao, C. Chen, B. Abidi, D. Page, A. Koschan, and M. Abidi. Sensor planning for automated and persistent object tracking with multiple cameras. In International conference on Computer Vision and Pattern Recognition (CVPR), 2008.

[26] H. Jin and G. Qian. Robust Multi-Camera 3D People Tracking with Partial Occlusion Handling. In International conference on Acoustics, Speech and Signal Processing, 2007.

[27] S. Khan and M. Shah. Consistent labeling of tracked objects in multiple cameras with overlapping fields of view. IEEE Transactions on PAMI, 25(10):1355-1360, 2003. 
[28] C. Chen, Y. Yao, D. Page, B. Abidi, A. Koschan, and M. Abidi. Camera Handoff with Adaptive Resource Management for Multi-camera Multi-target Surveillance. In International conference on Advanced Video and Signal Based Surveillance, 2008.

[29] U. Erdem and S. Sclaroff. Optimal placement of cameras in floorplans to satisfy task requirements and cost constraints. In OMNIVIS Workshop, 2004.

[30] E. Horster and R. Lienhart. On the optimal placement of multiple visual sensors. In Fourth workshop in Video networks and sureveillance systems, 2006.

[31] J. Zhao, S. Cheung, and T. Nguyen. Optimal Camera Network Configurations for Visual Tagging. IEEE Journal of Selected Topics in Signal Processing, 2(4):464-479, 2008.

[32] Agent-vi. Video Analytic Systems.

[33] N. Krahnstoever, T. Yu, S. Lim, K. Patwardhan, and P. Tu. Collaborative real-time control of active cameras in large scale surveillance systems. In Workshop on Multicamera and Multi-modal Sensor Fusion Algorithms and Applications, 2008.

[34] A. del Bimbo and F. Pernici. Distant targets identification as an on-line dynamic vehicle routing problem using an active-zooming camera. In VS-PETS, 2005.

[35] X. Zhou, R. Collins, T. Kanade, and P. Metes. A master-slave system to acquire biometric imagery of humans at distance. In ACM International Workshop on Video Surveillance, 2003.

[36] P. Chen, P. Ahammad, C. Boyer, S. Huang, L. Lin, E. Lobaton, M. Meingast, S. Oh, S. Wang, P. Yan, A. Yang, C. Yeo, L. Chang, and S. Sastry. Citric: A low-bandwidth wireless camera network platform. In International Conference on Distributed Smart Cameras (ICDSC), 2008.

[37] S. Hengstler, D. Prashanth, S. Fong, and H. Aghajan. MeshEye: A Hybrid-resolution smart camera mote for applications in distributed intelligent surveillance. In International conference on Information processing in sensor networks (IPSN), 2007.

[38] T. Teixeira, D. Lymberopoulos, E. Culurciello, Y. Aloimonos, and A. Savvides. A Lightweight Camera Sensor Network Operating on Symbolic Information. In ACM/IEEE Conference on Distributed Smart Cameras (ICDSC), 2006.

[39] BeagleBoard. Beagle Board System reference Manual Revision C 3.02009.

[40] R. I. Hartley and A. Zisserman. Multiple View Geometry in Computer Vision. Cambridge University Press, ISBN: 0521540518, second edition, 2004.

[41] David G. Lowe. Distinctive image features from scale-invariant keypoints. Int. J. Comput. Vision, 60:91-110, 2004.

[42] R. Hartley and A. Zisserman. Multiple View Geometry in Computer Vision. Cambridge University Press, 2000. 
[43] A. Fischler and C. Bolles. Random sample consensus: a paradigm for model fitting with applications to image analysis and automated cartography. Communications of ACM, 24, June 1981.

[44] D. Mills. RFC 1305: Network Time Protocol, 1992.

[45] T. Schmid, P. Dutta, and m. Srivastava. High-resolution, low-power time synchronization: an oxymoron no more. In International Conference on Information Processing in Sensor Networks (IPSN), 2010.

[46] R. Singh, M. Vatsa, A. Ross, and A. Noore:. A Mosaicing Scheme for Pose-Invariant Face Recognition. IEEE Transactions on Systems, Man, and Cybernetics, 37(5):1212-1225, 2007.

[47] Kalyan Veeramachaneni, Lisa Osadciw, Arun Ross, and Nisha Srinivas. Decision-level fusion strategies for correlated biometric classifiers. Computer Vision and Pattern Recognition Workshop, 0:1-6, 2008.

[48] Ayman Abaza and Arun Ross. Quality based rank-level fusion in multibiometric systems, 2009. 